\title{
Identification of Rapid Oxygen Exchange Through Site-Dependent Cationic Displacements on $\mathrm{CeO}_{2}$ Nanoparticles
}

\author{
Ethan L. Lawrence ${ }^{1}$, Barnaby D.A. Levin ${ }^{1}$, Tara M. Boland ${ }^{1}$, Shery L. Y. Chang ${ }^{2}$, and Peter A. Crozier ${ }^{1}$ \\ 1. School for the Engineering of Matter, Transport and Energy, Arizona State University, Tempe AZ. \\ 2. LeRoy Eyring Center for Solid State Science, Arizona State University, Tempe, AZ.
}

$\mathrm{CeO}_{2}$ (ceria) and ceria-based materials have many applications in heterogeneous catalysis, largely due to their oxygen exchange properties [1]. For a range of applications, including fuel cells, the oxygen exchange reaction is often the rate limiting step and it is critical to develop an atomic-level fundamental understanding of the reaction pathways. Under reducing conditions, ceria releases oxygen through the formation of an oxygen vacancy coupled with a cation transition from $\mathrm{Ce}^{4+}$ to $\mathrm{Ce}^{3+}$. Moreover, a lattice expansion ( $\sim 0.2-0.3 \AA$ ) occurs once oxygen vacancies are created on ceria surfaces [2]. The relative ease with which oxygen vacancies are created/annihilated on $\mathrm{CeO}_{2}$ is strongly dependent on particle surface and size and is an important catalytic property of ceria nanoparticles [3]. Aberration-corrected highresolution transmission electron microscopy (HRTEM) has been used to image atomic surface structures of $\mathrm{CeO}_{2}$ nanocubes with both $\mathrm{Ce}$ and $\mathrm{O}$ atomic columns clearly visible [4]. In situ environmental transmission electron microscopy (ETEM) can be used to observe dynamical processes that occur on ceria surfaces in reducing/oxidizing environments. Thus, atomic-level studies of $\mathrm{CeO}_{2}$ surfaces may enable the reaction pathways and active sites to be determined for oxygen exchange reactions, a fundamental outstanding problem in heterogeneous catalysis.

An aberration-corrected FEI Titan ETEM equipped with a Gatan K2 IS direct detection camera (with high detection quantum efficiency) was used to image $\mathrm{CeO}_{2}$ nanocubes at 40 frames/second and $5000 \mathrm{e}^{-} /\left(\AA^{2} \mathrm{~s}\right)$. The image corrector of the microscope was tuned to an optimum negative $\mathrm{C}_{\mathrm{s}}$ condition to enhance contrast from weakly scattering oxygen atomic columns. Images were aligned and binned to 10 frames/second to increase signal-to-noise for image processing. MIPAR software and MATLAB codes were used to determine centroid positions and integrated intensities of each $\mathrm{Ce}$ atomic column in each frame [5]. The root-mean-square-displacement (RMSD) of each atomic column was calculated to quantify the magnitude of displacement of each surface site. (A theoretical framework based on molecular dynamics, density functional theory, and image simulation for modeling structural and image motifs associated with oxygen exchange processes is discussed in [6]).

Figure 1a) shows a HRTEM image of a sum of 10 frames (1 sec. exposure) of a small (111) facet of a $\mathrm{CeO}_{2}$ cube in [110] projection in vacuum. Several step edge and corner atoms appear blurred relative to terrace atomic columns as indicated by the white arrows overlaid on the image. The centroid position of each atomic column was determined in each 0.1 second frame and Figure 1b) displays the tracks of displacement positions in each frame overlaid on the summed image. In Figure 1c), the calculated RMSD values are overlaid as colored circles according to the magnitude of RMSD. Due to small errors in image alignment and centroid fitting, an RMSD value of $\sim 5 \mathrm{pm}$ was calculated for bulk sub-surface atomic columns, which appear to be stationary. Thus, we estimate the systematic error to be $\sim 5 \mathrm{pm}$. As shown in Figure 1c), most surface terrace sites appear stable, while a large degree of displacement $(\sim 0.2-0.3 \AA)$ was observed at step edge sites and corner sites. The oxygen vacancy formation energies $\left(\mathrm{E}_{\mathrm{f}}\right)$ at corners and step edges have been calculated to be lower than vacancy formation energies on terrace sites. Consequently, atomic columns shift positions as oxygen is readily removed from or inserted into the ceria 
lattice at low $E_{f}$ sites. These site-specific displacements of Ce atomic column positions suggest variation in local oxygen exchange processes, potentially enabling preferred oxygen exchange sites to be determined [7].

\section{References:}

[1] Gorte, R.J., AIChE Journal 56 (2010) p. 1126.

[2] Muhich, C.L., The Journal of Physical Chemistry C 121 (2017), p. 8052.

[3] Trovarelli, A. and Llorca, J., ACS Catalysis 7 (2017) p. 4716.

[4] Lin, Y. et al, Nano Letters 14 (2014), p. 191.

[5] Sosa, J.M. et al, Integrating Materials and Manufacturing Innovation 3 (2014), p. 10.

[6] Boland, T.M. et al, Microscopy \& Microanalysis (2018).

[7] We gratefully acknowledge support of NSF grant DMR-1308085, the use of ASU's John M. Cowley

Center for High Resolution Electron Microscopy and use of the K2 IS camera courtesy of Gatan.
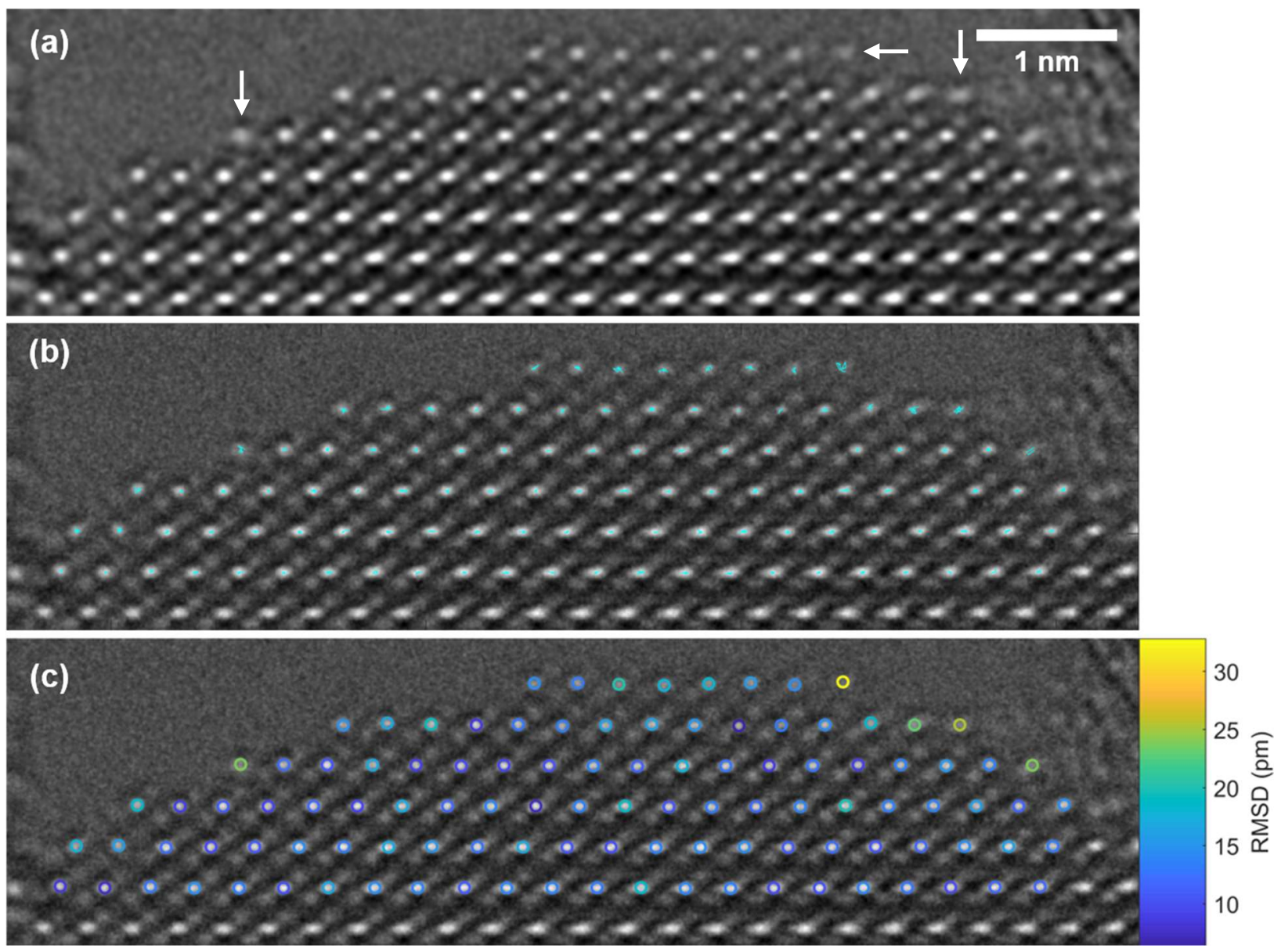

Figure 1. (111) surface of $\mathrm{CeO}_{2}$ (a) Sum of 10 frames (1 sec. exposure). Ce and $\mathrm{O}$ atomic columns visible at surface. (b) Ce atomic column centroid positions in each frame tracked in 10 frames overlaid on a summed image. (c) Root-mean-square displacements (RMSD) of each Ce atomic column position. 